\title{
THE IMPACT OF VR GRAPHICAL USER INTERFACE ON OCULUS TOUCH CONTROLLER AND OCULUS RIFT
}

\author{
Natchaphak Meeusah and Bennapa Pattanapipat \\ Multimedia Technology, Faculty of Mass Communication Technology, \\ Rajamangala University of Technology Thanyaburi, Thailand
}

\begin{abstract}
It is undeniably true that Virtual Reality (VR) has continuously been developed since 1800s and still have been produced till today. However, very few studies have attempted to study on the design of Virtual Reality Graphical User Interface (VR-GUI) that effectively empowers users to interact and immerse in a simulated world, via hardware and software with ease. Therefore, the aims of this research are to compare four different types of VR GUI Controller designs including (2D, 2D animation, 3D, and 3D animation) and to determine UI response time of the Oculus Touch Controller and compare the results with UI response time of Oculus Rift to determine what VR GUI is appropriate for which ages. 168 participants were purposely selected, aged from 12 to 17, 18 to 33, and 34 to 45. The experiment results showed that VR GUI had a significant impact on UI response time resulted from different types of VR GUI controllers. Last but not least, analysis of VR GUI controller user data had suggested that VR GUI developers should design appropriate VR GUI controllers that match all age groups in order for them to experience a fully immersive, perceptually real environment as quickly and efficiently as possible.
\end{abstract}

\section{KEYWORDS}

VR GUI, Oculus Touch Controller, Oculus Rift, Virtual Reality, Generation, Interactive.

\section{INTRODUCTION}

Across a broad range of disciplines and study fields, including training simulations, medicine, and education, Virtual Reality (VR) has been considered as one of the current and modern technologies being applied, as noted by [1]. With head-mounted display (HMD) technology as a display device, HMD has been created to provide users with more immersive virtual reality experience and affordable than ever before. Hence, as mentioned in the work of [2], there have been numerous research studies on interface usability of different types of user interface devices for controlling software in a virtual reality (VR) environment including Oculus Rift, Sony's PlayStation VR, and HTC Vive. Unfortunately, according to [2], very few studies have been focusing on VR GUI (Virtual reality graphical user interface) and thus different types of its VR GUI design should be introduced and compared for their further improvements, as stated by [3]. On top of that, VR GUI design guidelines and rules as specified by [4][5] should further be studied to help designers find universal standards of usability.

According to by [6] (Molina et al., 2003) VR GUI (Virtual Reality Graphic User Interface) has enabled a person to communicate with a VR Controller under the human direction through the operation of symbols, visual metaphors, and pointing devices. Hence, under good supervision of user, a great interface design would empower user to efficiently interact with the device and 
The International Journal of Multimedia \& Its Applications (IJMA) Vol.13, No. 6, December 2021

respond to their needs. In regard to the principle of VR GUI design [5][7] stated that its menu pattern could be displayed as follows 1) setting menu 2) main menu 3) play menu 4) information area and 5) display menu. As mentioned in the work of [8], in one hand the reason why developers prefer to design graphical user interface in virtual reality with 2 and 2.5-dimensional images is that it could create sense of perception and interpretation of the user for better understanding. For those users who are not acquainted with the interface design, on the other hand, may find issues particularly on misperceiving and misinterpreting of the data that are presented in 3 dimensional images in VR. Regarding to the VR controller, Head-Mounted Display and Interface, those users who wish to start using virtual reality device must get themselves accustomed to the VR controller in order for them to get controlled of their motion and movement. While HMD for VR is seen as supplemental to stimulate sensation of users and interface would help user understand the utility of controller, as stated by [9] in which same concept is also found in the utility guidance of keyboard, Nintendo Switch console, and PlayStation console, etc.

Therefore, the objectives of this work are to (i) make comparison between four different designs of VR GUI Controller or "Virtual Reality Graphical User Interface Controller" including (2D, 2D animation, 3D, and 3D animation) presence in virtual reality and (ii) to determine user's UI response time information and their age distribution in response to usability of the Oculus Touch Controller. The controller consists of a pair of left and right handheld units, each featuring an analog stick with five buttons that is compatible with the hardware for VR such as Oculus Rift. Unfortunately, with its possible complication of usage, Oculus Touch Controller's users might experience some difficulties in virtual reality. Therefore, the main focus of the research will be based on the study of VR GUI controller design that could eventually support user to best perceive immersive virtual environment while VR game developers could also make use of this research to help shape virtual reality devices and their applications in manufacturing industries.

\section{RELATED WORKS}

\subsection{Virtual Reality Graphical User Interface}

Graphic User interface (GUI) is a system of interactive visual components for computer software that could communicate with user via objects when interacts with them [5]. These graphical components are occasionally equipped with sounds, or visual effects. As mentioned by [5][7], the principle of GUI design tended be based on simplicity of interpretation thus good UI design should be able to allow user to control machines effectively and efficiently while interaction between the user and the product should be based on task completion, effectiveness, and satisfaction. To enhance UI design, informative images and characters including icons should be constructed for ease of user interpretation without time limit. In recent years, there have been literatures on UI including [10] who mentioned in his work that Product Interface Design method has followed Participatory Design approach which involve the user in the design process. This included the experiment of interface design for consumer appliances: microwave oven and a washing machine. Moreover, [11] has also reported in his comparative study on Non-diegetic interface and Diegetic interface design that when Oculus Rift as a Head-Mounted Display (HMD) was used to determine the efficacy of both interfaces, participants tended to choose Diegetic interface (3D) rather than Non-diegetic interface (2D) or other traditional menus due to its highest satisfaction and immersion. Additionally, during gameplay motion sickness was also not found. 
The International Journal of Multimedia \& Its Applications (IJMA) Vol.13, No. 6, December 2021

\subsection{Age Groups}

Because level of memory performance along with recognition conditions (i.e., recognizing of objects, surroundings, or sophistication of VR) could be differed among age groups, but particularly in elderly adults, who tend to possess reduced ability to reconstruct the spatiotemporal context as corresponded to the increase in critical false recognition occur with aging as mentioned in the findings of [12][13] research work and consequently, giving importance to design of VR GUI might have great impact on user recognition.

Concerning age group of users and their sensory-motor adaptation behavior in virtual reality environments, [14] revealed that subjects aged 8-12 years and aged 15-18 years responded to be unsuitable match between their motor behavior and the visual information; nonetheless, they generally presented distinctive prism adaptation behavior of a throwing after-effect error. Whilst, in the experimental study of [15] found that participants aged 15-18 were having similar results of cognitive development which affects spatial memory in virtual reality of participants aged

20-29. With these reasons, designing appropriate VR GUI would be instructional aids in classroom learning for younger age groups helping them enhance creative response in technological change. Hence, a study on designing feasible VR GUI for younger age group should also be considered. Besides having significant results of cognitive development in virtual reality environment in both groups, young age groups tangibly gained their VR benefits, in terms of anxiety and depression reduction during immersion of virtual reality as mentioned by [16]. Similarly, another distinctive VR benefit was also found in elderly adults when virtual reality memory trainings were implemented to help treat and control memory impairment as reported by [17].

\subsection{Oculus Rift and Controllers}

Regarding major technological development of virtual reality devices, numerous VR devices has been developed considerably including Kinect, Oculus Rift, Google Cardboard, Samsung Gear VR, HTC Vive, PlayStation VR, etc. These controllers specialized for creating fully immersive experience for VR users, have specifically been designed and enhanced by human-computer interface (HCI) as mentioned by [18].

Thus, [19] proposed their study on interaction accuracy of VR and VR controllers. In the study, relative position accuracy of Oculus Touch controller was found to be within an agreeable range and therefore is viable for tracking motion. [19] also mentioned that Oculus Touch controller was accurate, affordable and high in quality. In congruence, [20] supported in their works that Oculus Touch controller was one of the best choices to work with VR software while, [21] mentioned that Oculus Touch was as incredibly accurate as Kinect V1 and V2. Moreover, in a comparative research of [22], comparison of head tracking and the working area between Oculus Rift and the HTC Vive were studied in order to evaluate their accuracy and jitter experienced by users. Fortunately, results of the study were used for creating rehabilitation games and health-related software applications. Concerning the test results of comparison between HTC Vive and Oculus Rift, both devices showed excellent and comparable performance. When compared, Oculus Rift at sitting height had accuracy up to $1.5 \mathrm{~cm}$ and jitter $<0.5 \mathrm{~mm}$ and the HTC Vive presented accuracy and jitter at standing height (accuracy up to $1 \mathrm{~cm}$ and jitter $<0.35 \mathrm{~mm}$ ). However, [23] proposed that if Oculus Rift were to be as accurate as HTC, more sensors should be added.

Related to the studies, this research article aims to assess Oculus Touch Controller use ability by determining its UI response time of the Oculus Touch Controller and comparing its results with UI response time of Oculus Rift with four different types of VR GUI Controller designs including (2D, 2D animation, 3D, and 3D animation). 
The International Journal of Multimedia \& Its Applications (IJMA) Vol.13, No. 6, December 2021

\section{RESEARCH METHODOLOGY}

\subsection{Population and participants}

Meanwhile, the number of population of this research was 168 participants who were purposely selected. Participants were treated as homogenous in terms of their nationality which is Thai. The range of age is varied from 12-17, 18-33, and 34-45 years old. Concerning the user experience of Virtual Reality, all participants must not have any experiences in VR but having have First Person Shooters Games (FPS) experience is a must.
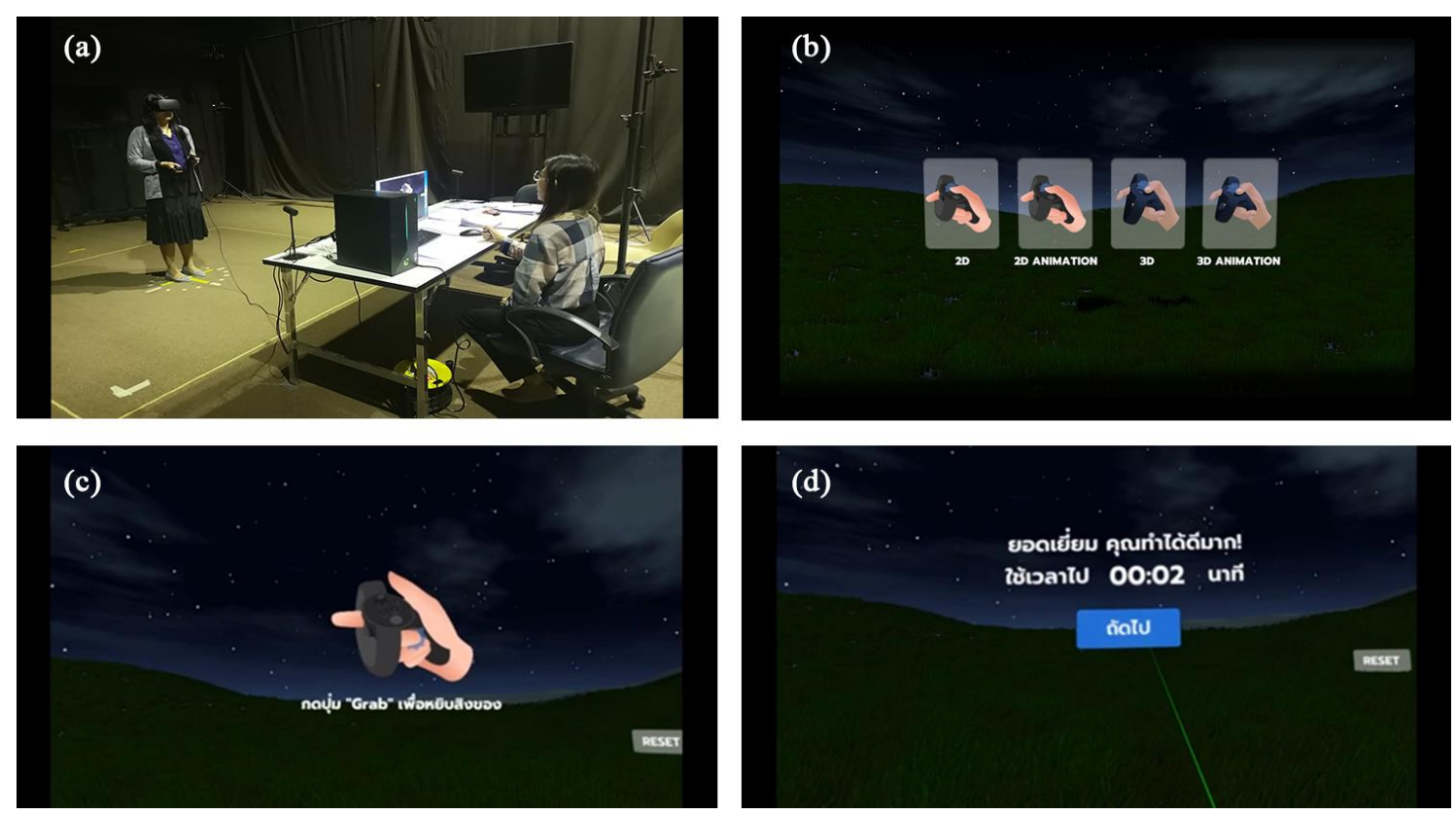

Figure 1. Photos of Immersive VR experience of user during the experiment, showing (a) Photo of the experiment, (b) Images of four types of VR GUI on the digital screen during the experiment, (c) Image representing VR GUI pressing button icon with description below,

(d) Image illustrating UI response time of participant's button press during the experiment

\subsection{Experimental design}

The objective of the experimental design is to make comparison of four VR GUI controller designs including (1) 2D, (2) 2D animation, (3) 3D, and (4) 3D animation. In (Fig.1-b), Design of press button guidance icon and appropriate hand position of Oculus Rift Touch controllers as shown in Fig.1-c) are illustrated. As mentioned in Fig.1-d, dependent variables of the experiment design including UI response time and user pressing each button are demonstrated. During the experiment, participants will be asked to interact to each design with VR GUI controller. 5 different buttons including (1) A Button (2) B Button (3) Grab Button (4) Trigger Button and (5) Joystick Button are found in the controller. When finishes conducting interaction testing under certain experimental conditions, participants will be asked to do their satisfaction rating on a scale of 1 to 5 ( 5 for strongly satisfied and 1 for strongly dissatisfied) for each design to complete an overall satisfaction questionnaire form without limitation of time. 
The International Journal of Multimedia \& Its Applications (IJMA) Vol.13, No. 6, December 2021

\subsection{Procedure}

Participants of the study are 168 subjects, 87 women and 81 men, aged between 12 and 17 years ( 30 women and $26 \mathrm{men} ; \mathrm{M}$ age $=41.1, \mathrm{SD}=3.31$, minimum $=35$, maximum $=45$ ), aged between 18 and 33 ( 35 women and 21 men; $\mathrm{M}$ age $=27.16, \mathrm{SD}=6.67$, minimum $=20$, maximum $=35)$, and aged between 34 and 45 years old $(\mathrm{M}$ age $=41.1, \mathrm{SD}=3.31$, minimum $=35$, maximum $=45$ ). In each age group, the researcher recruited an equal number of 56 participants. Prominently, each participant will initially be requested to interact with one VR GUI controller design with 3.11 minutes of interaction. Subsequently, another 5.13 minutes will be spent on participant interview making total experimental time of 8.23 minutes.

Prior to the experiment, participants will be demonstrated how to use the device. During the actual experiment, participants will be tested to determine their response time of each VR GUI controller design. The purpose of this experiment is to find out their perception of the designed VR GUI controller. In order to conduct the timing, VR GUI controller system will be required to show participant control over button press and when buttons of all Oculus Touch Controller are pressed correctly in accordance to the direction given by the system, the timing will then be stopped. Timing will be set in SI unit while all five buttons are set randomly to interact with participant. Additionally, all data of response time of user with VR GUI controller will be collected in the system. The range of participant age is varied from 12-17, 18-33, and 34-45 years old. 672 units of data of all 168 participants including their age, their response time of each button, and their interaction with all four controller designs are also collected.

Subsequently, still with Head-Mounted Display (HMDs) worn after experiment completion, participant will be asked to respond verbally to semi-ended questionnaire by rating their satisfaction on a scale of 1 to 5. Questions 1) "Design of 'hand icon' helps increase user visual understanding" 2) "Viewpoint of 'hand icon' helps increase user visual understanding 3) Design of 'Controller' helps increase user visual understanding" 4) "Viewpoint of 'Controller' helps increase user visual understanding" 5) "Placement of finger position helps increase user visual understanding" 6) Color blue suits call-to-action [CTA] button 7) Tooltip explaining the criteria for button is clear 8) Description font size of tooltip is clear 9) VR GUI controller of button icon design is clear and 10) VR GUI controller of button icon design is simple.

\section{Results}

\subsection{The comparison results of user response time between four VR GUI controller designs}

Were priory discussed. Subsequently, user response time of each axis Oculus Touch Controller joystick when compared to average response time of each VR GUI controller design were briefly discussed in the section. As shown in Figure 2, 
The International Journal of Multimedia \& Its Applications (IJMA) Vol.13, No. 6, December 2021

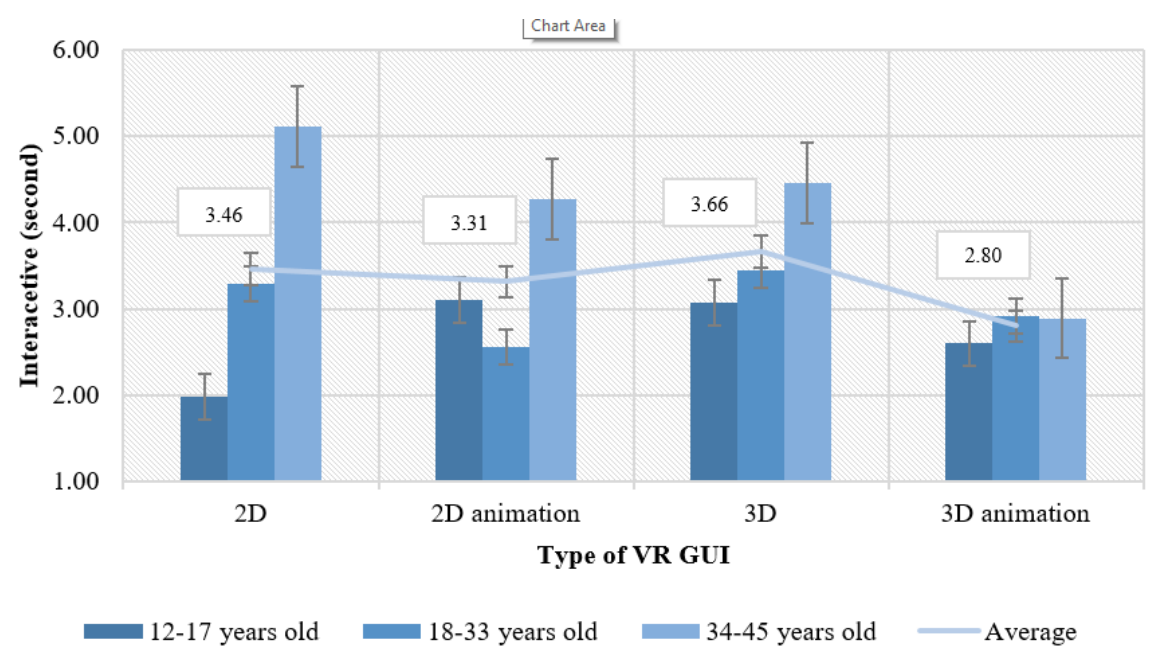

Figure 2. Comparison of average response time of each VR GUI controller design

Table 1. Comparison of average response time by VR GUI controller designs and range of age

\begin{tabular}{lcccc}
\hline $\begin{array}{l}\text { VR GUI } \\
\text { controller }\end{array}$ & $\begin{array}{c}\mathbf{1 2 - 1 7} \text { years old } \\
(\mathbf{n}=\mathbf{5 6})\end{array}$ & $\begin{array}{c}\mathbf{1 8 - 3 3} \text { years old } \\
(\mathbf{n}=\mathbf{5 6})\end{array}$ & $\begin{array}{c}\mathbf{3 4 - 4 5} \text { years old } \\
(\mathbf{n}=\mathbf{5 6})\end{array}$ & $\begin{array}{c}\text { Total } \\
(\mathbf{n}=\mathbf{1 6 8})\end{array}$ \\
\hline 2D & $\bar{x},($ S.D. $)$ & $\bar{x},($ S.D. $)$ & $\bar{x},($ S.D. $)$ & $\bar{x},($ S.D. $)$ \\
2D animation & $1.99,(0.71)$ & $3.29,(0.45)$ & $5.11,(0.78)$ & $\mathbf{3 . 4 6 , ( 0 . 6 4 )}$ \\
3D & $3.11,(0.10)$ & $2.56,(0.57)$ & $4.27,(0.69)$ & $\mathbf{3 . 3 1},(\mathbf{0 . 4 5})$ \\
3D animation & $3.08,(0.59)$ & $3.45,(0.44)$ & $4.46,(0.59)$ & $\mathbf{3 . 6 6 , ( 0 . 5 4 )}$ \\
\hline \multicolumn{1}{c}{ Total } & $2.60,(0.80)$ & $2.91,(0.68)$ & $2.89,(0.56)$ & $\mathbf{2 . 8 0 , ( 0 . 6 8 )}$ \\
\hline
\end{tabular}

When compared the average response time of each VR GUI controller design, it was found that the quickest response time of VR GUI controller design was 3D motion with average time of 2.80 seconds while 2D motion design was 3.31 seconds, 2D motionless design was 3.46 and 3D motionless design was 3.66 seconds.

In this study, One-Way Analysis of Variance will be applied to determine whether there is any statistical evidence that means of associated population are significantly different: ANOVA as described by [24] that the analysis tool could be used for analyzing the average variance of time interaction of each type of VR GUI, was applied. In addition, LSD (Fisher's Least Significant Difference) has also been applied to indicate confidence level at $95 \%$ or significant difference at $\mathrm{p}<.05$ as corresponding to that of the works of [25][26][27] who mentioned that LSD could be used for calculating the confidence level for all confidence intervals while it also could be used for controlling the individual error rate to a significance level that the user specifies. 
The International Journal of Multimedia \& Its Applications (IJMA) Vol.13, No. 6, December 2021

Table 2. Statistical comparisons of average response time of four VR GUI controller designs

\begin{tabular}{llrrrrr}
\hline & Mean & $\begin{array}{c}\text { Std. } \\
\text { Error }\end{array}$ & Sig. & $\begin{array}{r}\text { Lower } \\
\text { Bound }\end{array}$ & $\begin{array}{r}\text { Upper } \\
\text { Bound }\end{array}$ \\
\hline 2D & 2D Animation & .285 & 1.617 & .860 & -2.907 & 3.479 \\
& 3D & -1.380 & 1.617 & .394 & -4.574 & 1.812 \\
& 3D Animation & 2.547 & 1.617 & .117 & -.646 & 5.741 \\
\hline 2D Animation & 2D & -.285 & 1.617 & .860 & -3.479 & 2.907 \\
& 3D & -1.666 & 1.617 & .304 & -4.860 & 1.527 \\
& 3D Animation & 2.261 & 1.617 & .164 & -.931 & 5.455 \\
\hline 3D & 1.380 & 1.617 & .394 & -1.812 & 4.574 \\
& 2D & 1.666 & 1.617 & .304 & -1.527 & 4.860 \\
& 2D Animation & $3.928 *$ & 1.617 & .016 & .734 & 7.122 \\
\hline 3D Animation & -2.547 & 1.617 & .117 & -5.741 & .646 \\
& 2D & -2.261 & 1.617 & .164 & -5.455 & .931 \\
& 2D Animation & $-3.928^{*}$ & 1.617 & .016 & -7.122 & -.734 \\
\hline & 3D & & & & & \\
\hline
\end{tabular}

* The mean difference is significant at the 0.05 level.

In Table 2, results of two-step testing procedure for pairwise comparisons (Post Hoc Test) could be interpreted that the average response time of VR GUI controller was one-pair different from the ones of 3D and 3D Animation VR GUI controller, having a statistically significant difference of 0.05 .

Thus, the 3D design of VR GUI controller tended to possesses higher response time of interaction when compared to the 3D Animation.

\subsection{Comparison results of response time from interaction according to age group}

The authors of this research article had analyzed the collected data of response time from interaction according to age group. The average response time from interaction according to age group are presented in Table 1.

After comparing average response time of interaction 3 age groups, it could be found that age group of 12-17 years had tendency of spending average time of response of interaction at 2.69 seconds, followed by 18 to 33 years old at 3.05 while 34 to 45 years were considered highest at 4.14 seconds.

At the later stage of statistical analysis, One-Way Analysis of Variance: ANOVA was applied to find out the pairwise comparisons using LSD (Fisher's Least Significant Difference) method. Last but not least, the analysis for significant difference indication at $p<.05$ was also determined. 
The International Journal of Multimedia \& Its Applications (IJMA) Vol.13, No. 6, December 2021

Table 3. Comparison results of One-Way Analysis of Variance of average response time from interaction among age groups

\begin{tabular}{ccccccc}
\hline & & Sum of Squares & df & Mean Square & F & Sig. \\
\hline Generation & Between Groups & 56.470 & 2 & 28.235 & 14.684 & .00 \\
& Within Groups & 317.27 & 165 & 1.923 & & \\
\hline & Total & 373.741 & 167 & & & \\
\hline
\end{tabular}

* The mean difference is significant at the 0.05 level.

In Table 3, it was found that different response time from interaction of each age group had significant differences at $\mathrm{F}=14.684, \mathrm{Sig}>.000$.

After testing the hypothesis, One-Way Analysis of Variance testing of average response time from interaction among age groups had revealed that response time from interaction of each age group had a statistically significant difference of 0.05 .

In Table 4, post-hoc comparisons of response time from interaction of each age group (without concern for types of VR GUI) are shown as follows:

First pair (age group of 12-17 years old and age group of 34-45 years old) has statistically significant difference at 0.05 . Second pair (age group of 18-33 years old and age group of 34-45 years old) has statistically significant difference at 0.05 .

It could be assumed that response time interaction of participants age group of 34 to 45 years old with Oculus Touch Controllers was higher than those of 18 to 33 years old and 12 to 17 years old.

Table 4. Pairwise comparisons of response time from interaction of each age group are shown

\begin{tabular}{lcccccr}
\hline & & Mean & Std. Error & Sig. & $\begin{array}{r}\text { Lower } \\
\text { Bound }\end{array}$ & $\begin{array}{r}\text { Upper } \\
\text { Bound }\end{array}$ \\
\hline 12-17 years old & 18-33 years old & -2.821 & .262 & .283 & -.799 & .235 \\
& 34-45 years old & $-1.346^{*}$ & .262 & .000 & -1.863 & -8.829 \\
\hline 18-33 years old & 12-17 years old & .282 & .262 & .283 & -.235 & .799 \\
& 34-45 years old & $-1.064^{*}$ & .262 & .000 & -1.581 & -5.546 \\
\hline $34-45$ years old & $12-17$ years old & $1.346^{*}$ & .262 & .000 & .829 & 1.863 \\
& 18-33 years old & $1.064^{*}$ & .262 & .000 & .546 & 1.581 \\
\hline
\end{tabular}

* The mean difference is significant at the 0.05 level.

\subsection{Comparison results of user response time from interaction of Oculus Touch Controllers}

Data collected from user response time from interaction of each button of Oculus Touch Controllers was analyzed. Average user response time from interaction of each type of VR GUI is shown in Figure 3. 


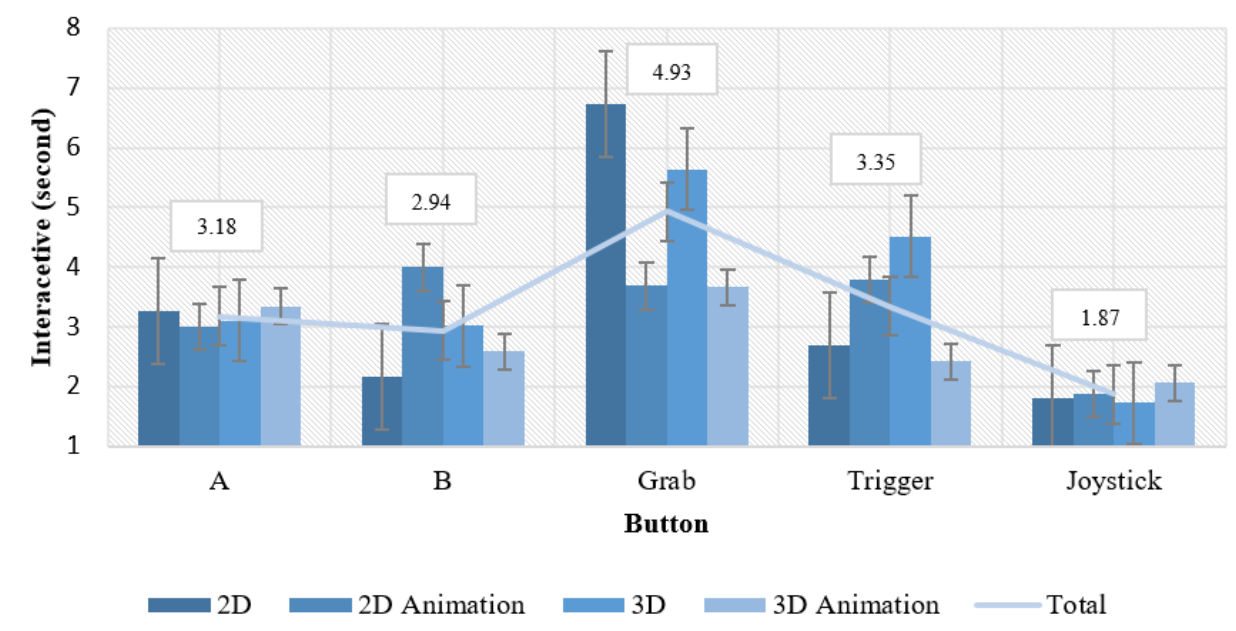

Figure 3. Comparison of user response time from interaction of Oculus Touch Controllers of each type of VR GUI

The comparison of average user response time from interaction on Oculus Touch Controllers showed that "Joystick" had the least amount of interaction with the average time of 1.87 seconds while "B" button had the average time of 2.94 seconds and the highest amount of interaction was "Grab" with the average time of 4.93 seconds.

\subsection{Feedback from research participants regarding to 10 questions of four types of user interface designs for VR GUI controller}

The answers were concluded as the average customer satisfaction score according to GUI controller types and age groups as shown in Figure 4.

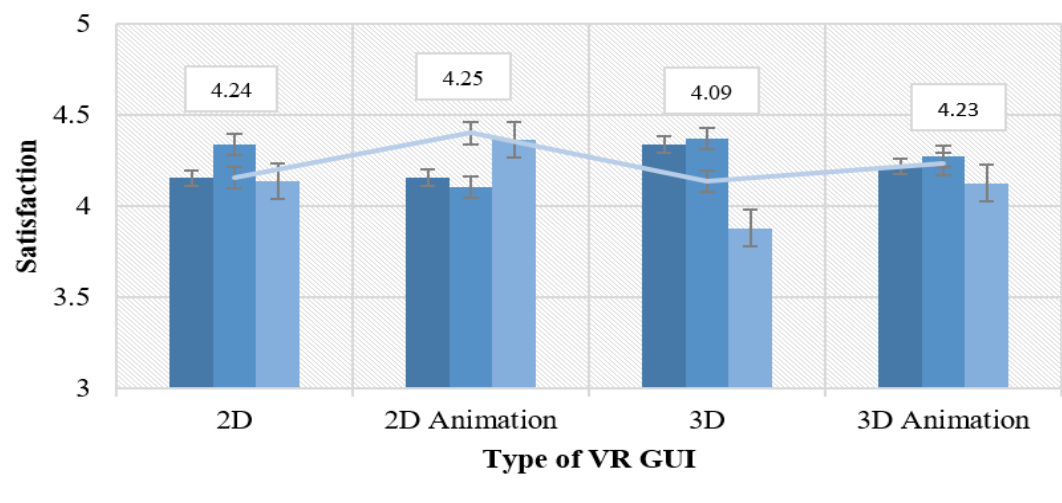

12-17 years old $\square$ 18-33 years old $\quad 34-45$ years old — Average

Figure 4. Average customer satisfaction score of research participants according to VR GUI designs and age groups

As illustrated in Figure 4, comparison results of feedback from research participants regarding to four types of user interface designs for VR GUI controller revealed that 2D Animation showed the highest average customer satisfaction score of 4.25 followed by 3D Animation of 4.23, 2D of 4.24. The least average score of 4.09 was $3 \mathrm{D}$ design. 
The International Journal of Multimedia \& Its Applications (IJMA) Vol.13, No. 6, December 2021

\section{Discussion}

Concerning four different designs of VR GUI controller, the results revealed that the highest average user response time was 3D animation. As close to real, 3D animation design was comparable with Virtual Reality, and able to create mental image as mentioned by (Bowman, 2013). 3D animation design was best appropriate message for conveying the right meaning to the users of 34-45 years old. The mentioned result also corresponded to the feedback from research participants describing that VR GUI was not problematic to understand and the buttons were easy to use. While 2D design tended to match users of 12 to 17 years old corresponding to results found in the research works of (Bowman, 2013) (Bowman et al., 2008) which revealed that most people were able to memorize and interpret well with 2 and $2.5 \mathrm{D}$. In addition, (Björling et al., 2019) also supported that these two types of design help teenagers deal with stress and anxiety. In contrast, [15] concluded from his research that both teenagers and adults had similar memory storage space. Moreover, 2D animation were suitable for age group of 18 to 33 years old while the slowest response time from interaction was 3D motionless. The result corresponded to the feedback of research participants that the design was too sophisticated to understand and difficult to make interpretation without any movements. Interaction via VR controller with $2 \mathrm{D}$ objects was found to be accurate but in case of eye-based interaction via eye -tracking technology with 3D objects, the interaction was most likely to be better according to the finding of [18].

After determining response time from interaction of 3 different age groups with VR GUI controller, it could be found that average response time of all age groups was 3.26 seconds. The least response time among from interaction was those who were 12 to 17 years old. The findings corresponded to the work of [14] describing how fast teenagers could adapt themselves to the application of VR. In contrast, the highest response time from interaction was those who were 34 to 45 years old with average time of 4.06 seconds. Regarding to the benefits of VR interaction, as mentioned by [28], those senior citizens who had problems with control of body movements, VR could increase sensory stimulation and improve body balance. Corresponding to the work of [12], findings revealed that in regard of navigation in VR, elderly adults were most likely to develop higher rate of recognition failure when compared to the younger ones. Therefore, having navigator system or protective system for recognition failure could allow elderly to possess faster recognition. Accordingly, VR GUI motion interaction, particularly in older adults, user interface design with perfect timing must be applied accurately in order to facilitate usability.

Considering the user interaction with Oculus Touch Controllers, average response time of controller buttons was 3.25 seconds. "Joystick" tended to possess least average user's response time of 1.87 seconds. As described in the feedback of research participants, "Joystick" had the clearest pressing button making it practical for use. In contrary, "Grab" button had the greatest response time from interaction of 4.93 seconds. When asked about "Grab" button, the research participants revealed that button was showing at the inappropriate position located at the lower part of Oculus Touch Controller and thus, when pressed with middle finger, the touch was taking too long to interact.

\section{Conclusions}

After comparing research participants' response time of Oculus Rift, the findings showed that each design of VR GUI had significant impact on response time from interaction statistically. Thus, 3D Animation design of VR GUI controller tended to possess the least response time of interaction meaning users could understand clearly and quickly. Regarding to the age group, different age groups created statistical difference of response time of interaction. Particularly with the age group of elderly, they seemed to have longer period of interaction while teenagers were 
The International Journal of Multimedia \& Its Applications (IJMA) Vol.13, No. 6, December 2021

shorter. Therefore, developers should be considering the position of Oculus Touch Controller buttons. Last but not least, "Joystick" button had the quickest user's response time but designing of "Grab" button should be avoided.

Recommendations for future research will be focused on design elements of VR GUI in order to find out the appropriate user response time and age group. In case developers would like to design VR GUI, age group should be considered in order to achieve highest satisfaction with VR technology.

\section{COMPLIANCE WITH ETHICAL STANDARDS}

Approved for ethical research involving human subjects

\section{REFERENCES}

[1] Zuckerberg, M. (2015) The future of connection. Presented at Facebook F8', Facebook's Developer conference, 25 March, 2015. Available at Internet: https://bit.ly/3CJqhcC [2021-02-11]

[2] Dorabjee, R., Bown, O., Sarkar, S., \& Tomitsch, M. (2015). Back to the future: identifying interface trends from the past, present and future in immersive applications. In Proceedings of the Annual Meeting of the Australian Special Interest Group for Computer Human Interaction (pp. 540-544).

[3] Bowman, D.A. (2013). 3D user Interfaces Bruno, F., \& Muzzupappa, M. (2010). Product interface design: A participatory approach based on virtual reality. International journal of human-computer studies, 68(5), 254-269.

[4] Norman, D. A. (2010). Natural user interfaces are not natural. interactions, 17(3), 6-10.

[5] Fröjdman, S. (2016). User experience guidelines for design of virtual reality graphical user interfaces controlled by head orientation input. Bachelor Degree Project in Cognitive Science, University of Skövde, Sweden (pp. 58).

[6] Molina, J. P., González, P., Lozano, M. D., Montero, F., \& López-Jaquero, V. (2003). Bridging the gap: developing 2D and 3D user interfaces with the IDEAS methodology. In International Workshop on Design, Specification, and Verification of Interactive Systems. Springer, Berlin, Heidelberg, (pp.303-315)

[7] Nielsen, J. (1995) Ten Usability heuristics. Nielsen Norman Group [website], January 1, 1995. Available at Internet: https://bit.ly/3jDwQ8d [2021-02-11]

[8] Bowman, D.A ., Coquillart, S., (2008) 3D user interfaces: new directions and perspectives. IEEE Computer Graphics and Applications, 28(6), 20-36.

[9] Seibert, J., \& Shafer, D. M. (2018). Control mapping in virtual reality: effects on spatial presence and controller naturalness. Virtual Reality, 22(1), 79-88.

[10] Bruno, F., \& Muzzupappa, M. (2010). Product interface design: A participatory approach based on virtual reality. International journal of human-computer studies, 68(5), 254-269.

[11] Salomoni, P., Prandi, C., Roccetti, M., Casanova, L., \& Marchetti, L. (2016, January). Assessing the efficacy of a diegetic game interface with Oculus Rift. In 2016 13th IEEE Annual Consumer Communications \& Networking Conference (CCNC) (pp. 387-392). IEEE.

[12] Sauzéon, H., N'Kaoua, B., Arvind Pala, P., Taillade, M., \& Guitton, P. (2016). Age and active navigation effects on episodic memory: a virtual reality study. British Journal of Psychology, 107(1), 72-94.

[13] Plancher, G., Gyselinck, V., Nicolas, S., \& Piolino, P. (2010). Age effect on components of episodic memory and feature binding: A virtual reality study. Neuropsychology, 24(3), 379.

[14] Adams, H., Narasimham, G., Rieser, J., Creem-Regehr, S., Stefanucci, J., \& Bodenheimer, B. (2018). Locomotive recalibration and prism adaptation of children and teens in immersive virtual environments. IEEE transactions on visualization and computer graphics, 24(4), 1408-1417.

[15] Narasimham, G., Adams, H., Rieser, J., \& Bodenheimer, B. (2020). Encoding Height: Egocentric Spatial Memory of Adults and Teens in a Virtual Stairwell. In ACM Symposium on Applied Perception 2020 (pp. 1-8). 
The International Journal of Multimedia \& Its Applications (IJMA) Vol.13, No. 6, December 2021

[16] Björling, E. A., Cicero, R., Sankar, A., \& Sekar, A. (2019). Thought Disposal: Co-Designing a virtual interaction to reduce stress in teens. In Proceedings of the 18th ACM International Conference on Interaction Design and Children (pp. 562-567).

[17] Optale, G., Urgesi, C., Busato, V., Marin, S., Piron, L., Priftis, K., ... \& Bordin, A. (2010). Controlling memory impairment in elderly adults using virtual reality memory training: a randomized controlled pilot study. Neurorehabilitation and neural repair, 24(4), 348-357.

[18] Hou, W. J., \& Chen, X. L. (2021). Comparison of Eye-Based and Controller-Based Selection in Virtual Reality. International Journal of Human-Computer Interaction, 37(5), 484-495.

[19] Shum, L. C., Valdés, B. A., \& Van der Loos, H. M. (2019). Determining the accuracy of oculus touch controllers for motor rehabilitation applications using quantifiable upper limb kinematics: Validation study. JMIR Biomedical Engineering, 4(1), e12291.

[20] Jost, T. A., Nelson, B., \& Rylander, J. (2021). Quantitative analysis of the Oculus Rift S in controlled movement. Disability and Rehabilitation: Assistive Technology, 16(6), 632-636.

[21] Otte, K., Kayser, B., Mansow-Model, S., Verrel, J., Paul, F., Brandt, A. U., \& Schmitz-Hübsch, T. (2016). Accuracy and reliability of the kinect version 2 for clinical measurement of motor function. PloS one, 11(11), e0166532.

[22] Borrego, A., Latorre, J., Alcañiz, M., \& Llorens, R. (2018). Comparison of Oculus Rift and HTC Vive: feasibility for virtual reality-based exploration, navigation, exergaming, and rehabilitation. Games for health journal, 7(3), 151-156.

[23] Suznjevic, M., Mandurov, M., \& Matijasevic, M. (2017, May). Performance and QoE assessment of HTC Vive and Oculus Rift for pick-and-place tasks in VR. In 2017 Ninth international conference on quality of multimedia experience (QoMEX) (pp. 1-3). IEEE.

[24] Fisher, R. A. (1992). Statistical methods for research workers. In Breakthroughs in statistics Springer, New York, NY. (pp.66-70).

[25] Williams, L. J., \& Abdi, H. (2010). Fisher's least significant difference (LSD) test. Encyclopedia of research design, 218, 840-853.

[26] Allcoat, D., \& von Mühlenen, A. (2018). Learning in virtual reality: Effects on performance, emotion and engagement. Research in Learning Technology, 26.

[27] Bhagat, K. K., Liou, W. K., \& Chang, C. Y. (2016). A cost-effective interactive 3D virtual reality system applied to military live firing training. Virtual Reality, 20(2), 127-140.

[28] de Bruin, E. D., Schoene, D., Pichierri, G., \& Smith, S. T. (2010). Use of virtual reality technique for the training of motor control in the elderly. Zeitschrift für Gerontologie and Geriatrie, 43(4), 229234. 